\title{
An Unusual Case of Endocarditis: Isolated Pulmonary Valve Endocarditis in Patient With Patent Ductus Arteriosus
}

\author{
Recep Tekin ${ }^{\mathrm{a}, \mathrm{f}}$, Halit Acet ${ }^{\mathrm{b}}$, Faruk Ertas ${ }^{\mathrm{c}}$, Rojbin Ceylan Tekin ${ }^{\mathrm{d}}$, Bedri Albudak
}

\begin{abstract}
Right-sided heart endocarditis is uncommon, comprising only $5-10 \%$ of all infective endocarditis cases. The majority of cases involve tricuspid valve. Isolated pulmonary valve endocarditis is very rare. As previously reported the ratio was 1.5 to $2.0 \%$ in all endocarditis and less than 90 cases of pulmonary valve endocarditis. This report presented a 9-year-old girl with isolated pulmonic valve endocarditis with patent ductus arteriosus.
\end{abstract}

Keywords: Infective endocarditis; Pulmonary valve; Vegetation; Patent ductus arteriosus

\section{Introduction}

Right-sided endocarditis is uncommon, comprising only $5-10 \%$ of all infective endocarditis cases [1]. The majority of cases involve tricuspid valve. Pulmonary valve endocarditis is a rare disease, usually associated with congenital heart disease, especially with patent ductus arteriosus (PDA). The ratio is 1.5 to $2.0 \%$ in all endocarditis [2]. Isolated pulmonary valve endocarditis is very rare, with less than 90 cases

\footnotetext{
Manuscript accepted for publication March 13, 2012

${ }^{a}$ Department Clinical Microbiology and Infectious Diseases, Dicle University Faculty of Medicine, Diyarbaklr, Turkey

${ }^{\mathrm{b}}$ Department of Cardiology, Diyarbaklr Training and Research

Hospital, Diyarbaklr,Turkey

${ }^{c}$ Department of Cardiology, Dicle University Faculty of Medicine, Diyarbaklr, Turkey

${ }^{\mathrm{d}}$ Department of Radiology, Diyarbaklr Children's Hospital, Diyarbaklr, Turkey

${ }^{\mathrm{e}}$ Department of Cardiology, Diyarbaklr Children's Hospital, Diyarbaklr, Turkey

${ }^{\mathrm{f}}$ Corresponding author: Recep Tekin, Department of Infectious

Diseases, Faculty of Medicine of Dicle University, Yenisehir 21280

Diyarbaklr, Turkey. Email: rectek21@hotmail.com

doi: http://dx.doi.org/10.4021/jmc600w
}

of pulmonary valve endocarditis being previously reported [3]. We reported a case with pulmonary valve endocarditis with patent ductus arteriosus.

\section{Case Report}

A 9-year-old female patient was admitted to the hospital with a high fever that continued for three-days. She had recently developed chills, chest pain, cough and shortness of breath. In admission, the patient's fever was $38.3{ }^{\circ} \mathrm{C}$. White blood cell count was $12,400 / \mathrm{mm}^{3}$, erythrocyte sedimentation rate (ESR): $140 \mathrm{~mm} / \mathrm{h}$, CRP: $123 \mathrm{mg} / \mathrm{dL}$, creatinine: $0.9 \mathrm{mg} /$ dL. Arterial blood pressure was $100 / 70 \mathrm{mmHg}$, pulse rate was 112 beats/min. In Physical examination she has hyperdynamic precordium and continuous "machinery" murmur above the pulmonic region consistent with patent ductus arteriosus. There was also hepatomegaly $(4 \mathrm{~cm}$ below right midcostal area) and splenomegaly $(2 \mathrm{~cm}$ below left costal area). Echocardiography showed large echodense and very mobile multiple vegetations on the pulmonary valve, hyperechogenic vegetations was attached to the pulmonary arterial wall (Fig. 1A). Left ventricular function was normal (ejection fraction: 65\%), specifically looked for PDA (Fig. 1B). There was not any vegetation on other valves (Fig. 1C).

She was admitted in intensive care unit and was started on intravenous ampicillin and gentamycin as empirically for infective endocarditis (Respect to Duke Criteria, infective endocarditis was confirmed and full dose antibiotic therapy was started). Three tubes blood samples were taken from different veins for blood culture. Streptococcus Viridans was positive in all three blood samples. On the third day, the blood cultures revealed vancomycin and gentamycinsensitive Streptococcus Viridans. Blood cultures revealed Streptococcus Viridans that was sensitive only to vancomycin and gentamycin. Vancomycin and gentamycin therapy was continued for 28 days. After repeated blood cultures became sterile at the 28 day of antibiotic therapy, the patient was discharged. In control echocardiography, there was not vegetation on pulmonary valve and main pulmonary artery (Fig. 2A, B). She has been referred to another center for PDA closure. 


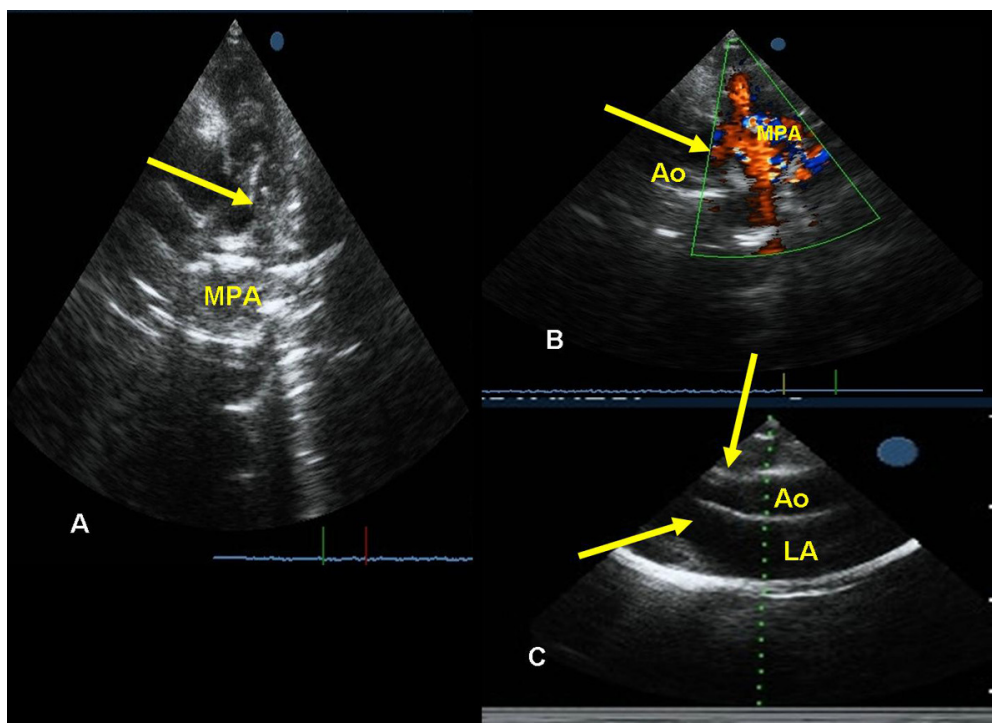

Figure 1. A: Transthoracic parasternal short axis views vegetations on pulmonary valve (arrow). B: Transthoracic parasternal short axis color Doppler imaging revealed PDA with left to right shunt (arrow). C: Transthoracic parasternal long axis views mitral and aortic valves (arrows).

\section{Discussion}

Pulmonary valve endocarditis is extremely rare, the ratio is only 1.5 to $2.0 \%$ in all hospital admissions for infective endocarditis [2]. The majority of right-sided heart infective endocarditis cases involve tricuspid valve. Infection may be isolated to the pulmonic valve alone or may concomitantly affect the mitral or aortic valve $[2,4]$. Both structurally normal and abnormal heart valves have been associated with pulmonic valve endocarditis $[2,4]$. The low incidence of in- fection on the pulmonic valve compared with other cardiac valves may relate to differences in hemodynamic pressures across the valves, oxygen saturation, underlying congenital or acquired valvular abnormalities, and the endothelial lining and relative vascularity of the valves.

Pulmonary valve endocarditis, even in the presence of structural heart disease, is rare with less than 90 cases of pulmonary valve endocarditis being previously reported [3]. The literature from 1960 to 2005 identified only 45 reported cases of pulmonary valve endocarditis in structurally normal

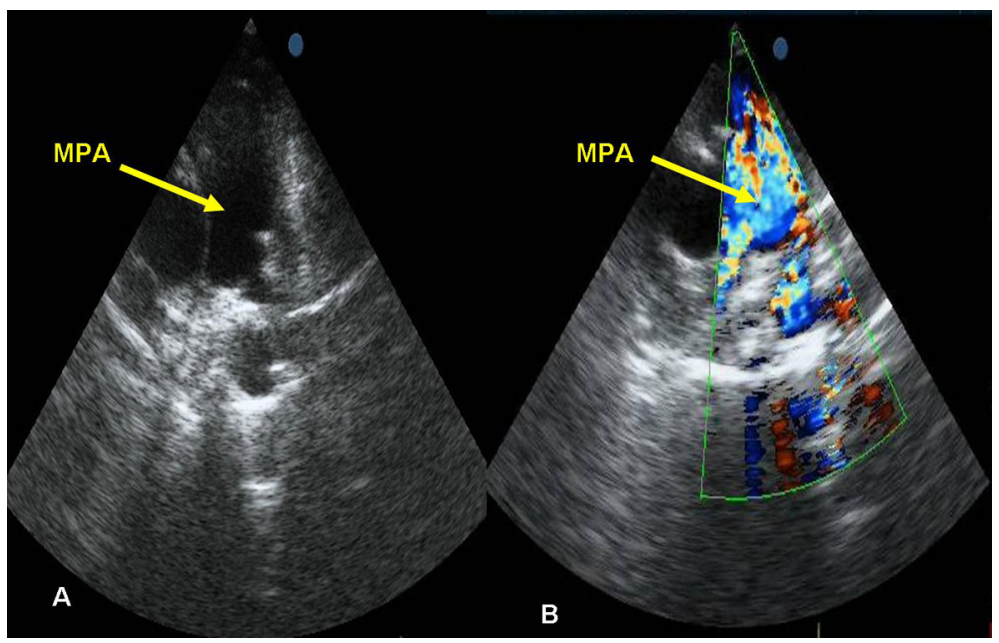

Figure 2. A: Transthoracic parasternal short axis views no vegetations on pulmonary valve and MPA (arrow). B: Transthoracic parasternal short axis views no vegetations on pulmonary valve and MPA color Doppler imaging revealed (arrow). Ao: aorta. MPA: main pulmonary artery, PDA: patent ductus arteriosus, LA: Left atrium. 
hearts [5]. Majority of isolated pulmonary valve endocarditis occurs in patients with congenital heart disease [2] as in our case. In this case, isolated pulmonary valve endocarditis could have initiated by the jet or turbulent flow created by PDA. During the preantibiotic era, infective endocarditis (IE) was a fatal complication of PDA and the common cause of death (42-45\%) in patients with PDA was IE [6]. with ongoing improvements in diagnostic tools, especially in echocardiography, antibiotic therapy, and surgery, the risk of IE in patients with PDA seems to have declined [7]. Although two-dimensional echocardiography shows a great value in the diagnosis of patients with IE, echocardiographic detection of vegetation within the pulmonary artery is extremely rare [8]. Documentation of endocarditis with a clinically silent PDA implies that the risk of endarteritis with a PDA may not be dependent on size [8].

The vast majority of infections were community-acquired. Predisposing factors include intravenous drug abuse $(28 \%)$, alcoholism (13\%), sepsis (7\%), central line infection $(7 \%)$ or other catheter-related infection $(5 \%)$, gonorrhea $(5 \%)$, dental extraction $(2.6 \%)$, bowel surgery $(2.6 \%)$, liver or renal transplantation $(2.6 \%)$, and colonic angiodysplasia (2.6\%) [9]. In $28 \%$ of cases, there was not identified predisposing factor. Compared with left-sided heart involvement, pulmonic valve infection tends to affect younger patients, and more than $80 \%$ of affected subjects are male. This case was female and had not any predisposing factors for IE.

It is difficult to diagnose without suspicion, since the first clinical manifestations often mimic pulmonary infection caused by septic pulmonary embolism. This delays appropriate treatment, thus careful physical examination and carefully getting medical history by physician are very important. Also, thorough echocardiographic evaluation of all cardiac valves, including right sided valves, should be carried out in all patients with suspecting infective endocarditis, especially when patient has a risk factor.

In 38 reviews, staphylococcus aureus was the most common microorganism detected in blood culture (44\%), in cases which has not pulmonic valvular abnormality before infection, followed by streptococci $(13 \%)$, streptococcus bovis $(5 \%)$, gonococcus $(5 \%)$, pseudomonas $(5 \%)$, E. coli $(5 \%)$, candida albicans $(5 \%)$, bacteroids fragilis (2.6\%), haemophilus influenza (2.6\%), and E.faecalis (2.6\%). Nonetheless, no organisms were cultured in $10 \%$ of the cases [10]. Streptococcus Viridans was positive in our case.

The clinical presentation of pulmonic valve endocarditis is similar with tricuspid valve infection. In this case, fever, shortness of breath, and pleuritic chest pain were predominated, and radiographic and laboratory findings corroborated the presence of pulmonary embolism. Approximately in onehalf of patients there is a pulmonic regurgitant murmur in cardiovascular examination. Because of nonspecificity of symptoms and the lack of typical peripheral findings that associated with left-sided mitral or aortic valve involvement, the diagnosis of pulmonic valve endocarditis may be delayed for up to 6 months. Parenteral antibiotic therapy is generally recommended for 4 to 6 weeks, indications for surgery are same as tricuspid valve, i.e. locally invasive infections, including abscess formation, progressive valve obstruction, incompetence, and relapsing infection despite full-dose antibiotic therapy. Compared to other right-sided heart valves infection, the prognosis of pulmonic valve endocarditis is generally better than mitral or aortic infection [2]. This case was treated 28 days successfully with antibiotic therapy and blood cultures remained sterile.

\section{Conclusion}

Pulmonic valve endocarditis is an extremely rare infection that has same epidemiologic, clinical, radiologic, microbiologic, and prognostic features with tricuspid valve endocarditis. In this report, we presented an unusual case of isolated native pulmonary valve endocarditis and pulmonary artery endarteritis in a child with congenital PDA. She was treated successfully with antibiotic therapy. Carefully evaluating pulmonic valve in echocardiography is very important, when vegetation is not detected in other valves and clinical suspicion for infective endocarditis is considered. After a review of the literature identified, less than 90 cases of pulmonary valve endocarditis were previously reported. Compared with the left-sided heart valves, the involvement of pulmonic valve infection tends to affect younger patients and more than $80 \%$ of affected subjects are normally male.

\section{Conflict of Interest}

None.

\section{Note}

This case report was accepted at poster presentation "4th EUROASIA CONGRESS OF INFECTIOUS DISEASES 'Clinical Microbiology, Enfectious Disease, Immunology and Epidemiology' Sarajevo, Bosnia and Herzegovina, June $1-5,2011 "$.

\section{References}

1. Chan P, Ogilby JD, Segal B. Tricuspid valve endocarditis. Am Heart J. 1989;117(5):1140-1146.

2. Ramadan FB, Beanlands DS, Burwash IG. Isolated pulmonic valve endocarditis in healthy hearts: a case report and review of the literature. Can J Cardiol. 2000;16(10):1282-1288.

3. Tariq M, Smego RA, Jr., Soofi A, Islam N. Pulmonic 
valve endocarditis. South Med J. 2003;96(6):621-623.

4. Nakamura K, Satomi G, Sakai T, Ando M, Hashimoto A, Koyanagi H, Hirosawa K, et al. Clinical and echocardiographic features of pulmonary valve endocarditis. Circulation. 1983;67(1):198-204.

5. Schroeder RA. Pulmonic valve endocarditis in a normal heart. J Am Soc Echocardiogr. 2005;18(2):197-198.

6. Bilge M, Uner A, Ozeren A, Aydin M, Demirel F, Ermis B, Ozkokeli M. Pulmonary endarteritis and subsequent embolization to the lung as a complication of a patent ductus arteriosus--a case report. Angiology. 2004;55(1):99-102.
7. Thilen U, Astrom-Olsson K. Does the risk of infective endarteritis justify routine patent ductus arteriosus closure? Eur Heart J. 1997;18(3):503-506.

8. Balzer DT, Spray TL, McMullin D, Cottingham W, Canter CE. Endarteritis associated with a clinically silent patent ductus arteriosus. Am Heart J. 1993;125(4):1192-1193.

9. Cassling RS, Rogler WC, McManus BM. Isolated pulmonic valve infective endocarditis: a diagnostically elusive entity. Am Heart J. 1985;109(3 Pt 1):558-567.

10. Johnson DH, Rosenthal A, Nadas AS. A forty-year review of bacterial endocarditis in infancy and childhood. Circulation. 1975;51(4):581-588. 